\title{
ESCOTOSIS PROFESIONAL EN ADICCIONES E IMPLICANCIAS ÉTICAS
}

\author{
PROFESSIONAL MENTAL BLINDNESS IN ADDICTIONS AND ITS ETHICAL IMPLICATIONS
}

Martín Nizama-Valladolid ${ }^{1, a}$

\section{RESUMEN}

Se describe el estado de escotosis en el médico y en los demás profesionales de la salud acerca del fenómeno adictivo (FA), definido como carencia de conocimiento científico idóneo sobre las adicciones, lo que les condiciona una actitud prejuiciada, dogmática y sesgada de esta enfermedad, con consecuencias devastadoras para el paciente, su familia y el entorno social. Además, se establece la incongruencia en la praxis profesional entre los aspectos bioéticos y clínicos de las adicciones. Se discute el tema de los derechos humanos del paciente y se fundamenta la procedencia de la firma de la autorización familiar del internamiento involuntario del paciente carente de conciencia de enfermedad. Finalmente se proponen algunas alternativas para afrontar la escotosis.

Palabras clave: "Ceguera" profesional en adicciones; Oscurantismo mental profesional, "Agujero negro" en adicciones (fuente: DeCS BIREME).

\begin{abstract}
This article describes professional mental blindness (Escotosis) in physicians and other health professionals regarding the addictive phenomena (AP) as a lack of suitable scientific knowledge about the addictive phenomenon that conditions the adoption of prejudiced, dogmatic and biased attitude about this disease with devastating consequences to the patient, his family and his social surroundings. It also establishes the inconsistency in professional practice between bioethics and clinical aspects of addictions. It discusses the subject of human rights of the patient and supports the involuntary hospitalization by the family without the informed consent of the patient who is not aware of his illness. Finally some alternatives to face this problem are proposed.
\end{abstract}

Key words: Professional blindness in addictions; Professional mental obscurantism; "Black hole" in addictions. (source: MeSH NLM).

\section{INTRODUCCIÓN}

El médico que desconoce el manejo holístico del fenómeno adictivo, cautivo de este sesgo cognitivo, cree que puede rehabilitar a un adicto avanzado, grave o terminal ${ }^{1}$, mediante el tratamiento ambulatorio, indicándole fármacos autoadministrados y una desintoxicación cada vez que el paciente asiste solo a su consulta, sin el indispensable acompañamiento de sus familiares ${ }^{2,3}$, quienes constituyen el verdadero eje terapéutico. Por otro lado, algunos especialistas valoran su fracaso terapéutico como

un desenlace normal y terminan por expulsar al paciente debido a la carencia de respuesta terapéutica ${ }^{4}$, racionalizándolo mediante la creencia errónea de que el paciente se ha resistido a colaborar; es decir que se ha negado a ejercer su "fuerza de voluntad", perpetuándose así el problema adictivo. Este proceso autodestructivo es evitable mediante programas de intervención sostenidos, conducidos por equipos profesionales con experticia en el afronte holístico del fenómeno adictivo.

\footnotetext{
'Facultad de Medicina, Universidad Peruana Cayetano Heredia. Lima, Perú. Facultad de Medicina, Universidad Nacional Mayor de San Marcos. Lima, Perú. Instituto Nacional de Salud Mental “Honorio Delgado- Hideyo Noguchi”. Lima, Perú.

aMédico Psiquiatra, Doctor en Medicina.
}

Correspondencia: Dr. Martín Nizama Valladolid. Dirección: Torres de Consultorios Anglo Americana Alfredo Salazar 314, Of. 303, Lima 27, Perú. Teléfono: 511 4219939. Anexo: 430 y 433. Correo : maniva@terra.com.pe 


\section{DEFINICIÓN}

Se define escotosis profesional como la percepción y conceptualización subjetiva de la enfermedad adictiva, que conduce a reacciones páticas inadecuadas ante el fenómeno adictivo por parte de determinados profesionales de la salud; sean estos médicos, enfermeras, trabajadores sociales o psicólogos, quienes abordan este grave problema de salud pública mediante intervenciones contaminadas por creencias y actitudes irracionales, verbi gratia, mitos, tabúes, prejuicios y comportamientos carentes de sustento científico y ético.

\section{DIAGNÓSTICO SITUACIONAL}

El profesional de la salud con escotosis conceptualiza las adicciones en forma simplista como un vicio, un trastorno de conducta o como un problema psicosocial; mas no como una enfermedad volitiva ${ }^{5,6,7}$, asumiendo erróneamente que el adicto puede cesar la conducta adictiva por sí mismo, si se lo propone. Por el contrario, el adicto, carente de conciencia de enfermedad, evita el tratamiento. Cuando el adicto simula aceptar un tratamiento, lo hace bajo sus condiciones. Su verdadero propósito es aplacar el acoso familiar ${ }^{8}$, superar conflictos laborales ${ }^{9}$ o acatar una orden policial o judicial. Durante el desarrollo del programa intenta sabotearlo y termina abandonándolo, lo cual con frecuencia acaba en un previsible desenlace funesto.

El profesional de la salud mental con escotosis en adicciones, considera equivocadamente que puede manejar esta enfermedad como los desórdenes mentales menores: ansiedad, depresión, fobia o estrés postraumático. Por eso exige a priori al adicto que posea conciencia de enfermedad y motivación para el tratamiento; debido a esto, rechaza la posibilidad de hospitalizar al paciente, excepto cuando éste lo solicita voluntariamente, lo cual es inviable, pues se han encontrado que la carencia de conciencia de enfermedad alcanza tazas del $72.6 \%$ en poblaciones alcohólica ${ }^{10}$ y que existe una relación clara entre el consumo de drogas y la inconciencia de la actitud hacia su búsqueda ${ }^{11}$. Finalmente, menos del $2 \%$ de adictos busca ayuda voluntariamente ${ }^{12}$. Por ello, el éxito terapéutico dependerá del apoyo que reciba el paciente por parte de su entorno familiar y social, mas no de su inexistente voluntad; puesto que ha sido abolida transitoriamente por la adicción.

Así mismo, este profesional cree en la falacia de que las adicciones son incurables y que muy poco o nada se puede hacer ante esta enfermedad. Incluso, existen especialistas que sugieren que adicciones como la dependencia al tabaco no deberían ser tratadas por ser una dependencia supuestamente menos perjudicial frente a otras formas ${ }^{13}$. Otros proponen a la familia que espere a que el enfermo "toque fondo" y que entonces él solicitará ayuda médica. Otras veces indica a la familia que expulse al adicto del hogar para que "reaccione" o para que se evite mayor sufrimiento. Debido a estos prejuicios, aquel profesional excluye en forma parcial o total a la familia del proceso terapéutico, limitando su intervención a un enfoque individualista centrado únicamente en el adicto, aún cuando las tazas de abandono en las terapias individuales como los programas con metadona, naltrexona o los programas libres de droga e incluso en las comunidades terapéuticas, son elevadas ${ }^{4}$.

Con este enfoque reduccionista no se asegura el éxito terapéutico a largo plazo, lo cual acarrea inexorables recaídas o abandonos con el consiguiente sufrimiento de la familia y de su entorno social. Al respecto Guajardo y Kushner (2004) ${ }^{14}$ expresan "Los trastornos adictivos han representado un serio desafío para psiquiatras y psicólogos, lo que ha llevado a un descrédito de los tratamientos tradicionales. Desde luego son muchos los pacientes que fracasaron en su intento de dejar la droga a través de psicoterapias individuales". Asimismo, Garrido, Jaén, y Domínguez (2004) ${ }^{15}$, acotan en referencia al rol de la familia en la ludopatía "a pesar de que el problema de juego se vive como un problema que afecta a la familia, (...) su papel como factor de mantenimiento o regulación de la conducta de juego patológico de uno de sus miembros no ha sido contemplado, por lo que habitualmente se utilizan estrategias de tipo conductual o cognitivo-conductual centradas en el propio individuo que presenta el síntoma. Es decir los modelos de tratamiento siguen utilizando el principio de que el juego es una conducta que se entiende sólo a partir de las características propias del individuo". Del mismo modo, debido a la escotosis se atribuye el origen de las adicciones a la unicausalidad o a una pluricausalidad restringida, sujeta a modelos de enfoque restrictivo, tales como: conductual, motivacional o cognitivo-conductual ${ }^{16}$; que toman como eje terapéutico al paciente y no a la familia.

Por su lado, la familia tarda en enterarse que uno o más de sus miembros son adictos. Y cuando final- 
mente esto ocurre, se resiste durante años a aceptar el problema y buscar ayuda especializada. En promedio una familia tarda en buscar atención Médica 4.69 años desde que percibe el consumo ${ }^{17}$. El entorno social extrafamiliar allegado usualmente logra percibir el problema ${ }^{18}$, lo que conduce a la familia llena de prejuicios, inicialmente a negar y luego a ocultar la adicción. Entonces, desde el inicio la familia presenta la coadicción o codependencia, que es el mantenimiento de la adicción por parte de la familia, mediante la permisividad, tolerancia y encubrimiento del patrón adictivo, dejándose manipular por el adicto, quien termina por esclavizarlos ("esclavos infelices"). La escotosis profesional imposibilita reconocer esta homeostasis patológica familiar, a la que se considera "víctima" de la conducta adictiva en lugar de ver el sistema familiar como uno de los factores que mantienen, potencian y favorecen la conducta patológica ${ }^{15}$. En suma, la familia también carece de conciencia de enfermedad, lo cual explica su demora en la búsqueda de la ayuda terapéutica oportuna. Así, la enfermedad se perpetúa agravándose severamente, con consecuencias funestas para el enfermo y su entorno familiar.

De otro lado, debido a la escotosis profesional se acepta una artificiosa clasificación de las adicciones: alcoholismo, tabaquismo, drogadicción y ludopatía (juego patológico como si se tratara de entidades nosológicas independientes, lo cual es una caricatura. Merced a esta clasificación, se cree que mientras el paciente no consuma, cocaína, marihuana o heroína; puede beber licor, fumar o automedicarse con psicofármacos. Se asume que la persona es adicta a una sola sustancia que por lo general termina siendo la ilegal, cuando en realidad el paciente presenta una poliadicción ${ }^{19}$; adicto a las drogas legales e ilegales, debido a que el circuito cerebral de la recompensa autogratificante $20,21,22$ no distingue entre sustancias lícitas e ilícitas. Inclusive, algunas organizaciones de salud evidenciando la escotosis que existe entre sus miembros, han creado el día anual para la celebración del "Día del No Fumador", 31 de mayo y el "Día Internacional de Lucha Contra las Drogas", 26 de Junio, como acciones totalmente independientes la una de la otra. Por el contrario, una clasificación que considere el abordaje terapéutico holístico debe agrupar las adicciones en cuatro categorías: químicas, electrónicas, lúdicas y sociales $^{23}$, las mismas que constituyen una sola enfermedad hedonista.

En el Perú y probablemente en varios países de
Latinoamérica, los familiares desesperados ante el fracaso terapéutico, endosan a sus pacientes a las Comunidades Terapéuticas (CT), algunas de ellas informales y otras clandestinas, por ser económicamente más accesibles. Estas CT informales o clandestinas son dirigidas por autodenominados "ex-adictos", que no han acreditado haber sido rehabilitados. Es más, en ellas se cultiva una actitud antimédica, según la cual, los médicos agravan las adicciones pues "drogan más a los adictos con pastillas" y, usan el eslogan de que "sólo un ex-adicto puede curar a un adicto"24. Además, es una verdad de perogrullo que aquellas $\mathrm{CT}$, tienen como único propósito el lucro anético, pues no existe evidencia de que las posibilidades terapéuticas reales incluso de las CT profesionales sean superiores frente a otras formas de tratamiento ${ }^{25}$. En estas instituciones, los adictos ingresan y egresan en forma libérrima, constituyéndose el círculo vicioso denominado "puerta giratoria". Los pacientes de esos "centros" aprenden el comportamiento disocial de sus pares y adquieren la institucionalitis, por la cual el adicto se torna en un avezado manipulador de sus "terapeutas" y se psicopatiza gravemente, convirtiéndose en una grave amenaza familiar y social.

En el ámbito de la promoción y de la prevención, el profesional con escotosis habitualmente no visualiza los factores protectores de la salud mental y espiritual; tampoco los factores de mantenimiento de la enfermedad adictiva: el entorno familiar, mercado hedonista, entorno social (principalmente el amical) y ecológico; factores que constituyen la base del Fenómeno Adictivo'. Dicho profesional adopta posiciones dogmáticas y cientificistas priorizando los enfoques reduccionistas, subestimando el entorno familiar, social y ecológico. Por este motivo, las "campañas antidrogas" han devenido en fracasos estrepitosos, observándose el incremento de millones de adictos en la sociedad global; con la consecuente desintegración de igual número de familias, y la estigmatización del adicto debido a su alta peligrosidad social.

\section{CAUSAS}

Las probables causas de la escotosis profesional son múltiples, principalmente:

- Vacíos académicos

- Dogmatismo cientificista

- Negligencia profesional

- Creencias irracionales 
- Conflicto de intereses

- Deformación profesional

- Estigmatización

- Social Falta de investigación

\section{CONSECUENCIAS}

\section{Recidiva}

Facilitación de la recurrencia adictiva mediante diversos actos conscientes o inconscientes de la familia. Entre ellos se encuentran: irresponsabilidad, ausencia de control, acceso a dinero fácil, frecuente exposición al riesgo adictivo, permisividad, tolerancia o creencia de que la persona ya se encuentra sana, tras períodos cortos de tratamiento. En suma, se debe principalmente a la ausencia o ineficacia del sostenimiento de la abstinencia total continua, por parte de un entorno familiar no comprometido con la recuperación del adicto a largo plazo.

\section{Fracaso terapéutico y deserción}

El fracaso terapéutico se objetiva en la ineficacia para alcanzar la abstinencia total permanente y la remisión de la conducta disocial del adicto, por la persistencia de los estilos de vida hedonistas en un entorno familiar disfuncional. El abandono del tratamiento es la norma en estos pacientes.

\section{"Puerta giratoria"}

Los adictos ingresan y egresan de los internamientos sin beneficio para su salud. Esta situación está determinada por la ineficacia de los tratamientos empíricos o clandestinos a los cuales se les expone. Los pseudo ex-adictos o los profesionales sin experticia en adicciones que intervienen en este embuste, lo hacen con fines meramente lucrativos. Con ello se institucionaliza la desesperanza familiar.

\section{Conveniencia Anética}

Es la asociación de intereses encubiertos que se establece entre el adicto, la familia y el terapeuta; por la cual el enfermo aparentemente accede a un tratamiento bajo las condiciones que él impone con el objetivo de aplacar la ira o hartazgo de los suyos. Por su lado, los familiares le costean dicha terapia condicionada, con el propósito de mitigar sus sentimientos de culpa. A su vez, el terapeuta toma el caso aceptando las condiciones que le impone el enfermo, con fines meramente lucrativos; lo hace a sabiendas que no es un especialista diestro en el manejo de estos enfermos disociales, activos mili- tantes de la antiterapia. De este modo, supuestamente, todos ganan y están contentos, aunque el adicto a la par que asiste a la terapia continúa desarrollando activamente su conducta adictiva; la familia se tranquiliza temporalmente y el terapeuta cobra honorarios por no curar. Un fraude consentido.

\section{Implicancias bioéticas}

En el campo de la bioética, la valoración de cada enfermo y su entorno familiar debe sustentarse en su condición clínica y no en prejuicios ni en posiciones ideológicas y dogmáticas adoptadas a priori. El profesional con escotosis al excluir o subestimar esta realidad clínica, estaría mostrándose indolente ante la autodestrucción inexorable del adicto. De igual modo, su escotosis le imposibilita comprender que la adicción hace tabla rasa de los principios fundamentales de la ética médica ${ }^{26}$.

\section{Autonomía}

La autonomía, cuyo fin último es la búsqueda del mayor bien personal (salud, bienestar, libertad y autorrealización), pierde su valor esencial. El adicto debido a su enfermedad, ha perdido esta capacidad ética. Por el contrario, al carecer de conciencia de enfermedad y tener anulada la voluntad para ejercer responsablemente su libre albedrío, se convierte en dependiente de "algo" cuya necesidad le produce un deseo incontrolable de disfrutarlo, seguido del culposo sufrimiento postconsumo. Así, pierde el sentido de vida, careciendo de proyecto de autorrealización personal.

\section{Beneficencia}

Procurar el mayor beneficio y benevolencia en favor de la persona. Este principio también se desnaturaliza al permitírsele al adicto optar "libremente" por el no tratamiento, se le condena a la degradación progresiva de sí mismo, poniendo en riesgo su integridad individual, su vida y la de su familia.

\section{No maleficencia}

Primum non nocere, primero no hacer daño. El médico con su pasividad expone al enfermo justamente a los efectos malignos de la enfermedad volitiva; vale decir al "suicidio dulce", lo cual ocurre de modo insidioso, progresivo, inconsciente e inexorable.

\section{Justicia}

Procura el bien común. Sin embargo, debido a la inacción médica, la familia se expone a la sujeción por parte del adicto; puesto que las consecuencias de las adicciones son gravemente perniciosas para 
ella y el dependiente. La familia, primero se enferma, luego se desorganiza y finalmente se desintegra, sufriendo el apocalipsis familiar. También tiene impacto disruptivo sobre el entorno social. Es importante enfatizar que cuando surge conflicto entre el principio de autonomía y el principio de justicia, debe primar el segundo.

\section{Derechos humanos}

Según el artículo 3 de la Declaración de los Derechos Humanos ${ }^{27}$, el primer derecho humano fundamental es el derecho a la vida; $y$ establece con prioridad la libertad personal y la seguridad de su persona. Entonces, si la adicción precisamente arrasa estos derechos inherentes a la persona humana, ¿Cómo ignorar estos derechos primarios en una persona cuya enfermedad le ha abolido precisamente la voluntad, el libre albedrío y la autonomía? Es más, en virtud de la "ética de mínimos" se considera que los principios de no maleficencia y justicia como de primer nivel ${ }^{28}$. Ergo, los derechos humanos de cada miembro de la familia y de las personas del entorno deben ser respetados. Sin embargo, el sesgo dogmático, cientificista y legal sólo se circunscribe a los derechos del adicto, en perjuicio de su entorno familiar y social, lo cual es incoherente y cruel. El ser humano integra el tejido social y no es una torre de marfil.

\section{Autorización de la familia}

Es la responsabilidad que asume la familia al firmar la Autorización de Internamiento indicado por el médico, en representación del paciente, mientras él se encuentre incapacitado temporalmente, debido a su inconsciencia de enfermedad. Este procedimiento se sustenta en el vínculo de pertenencia al grupo familiar, su relación de consaguinidad y el vínculo afectivo y espiritual que los liga por siempre.

Autorizar una intervención terapéutica en uso de la plena libertad individual, es una facultad inherente a la persona con integridad mental, espiritual y física; más no, de aquella persona que debido a su inconsciencia de enfermedad, carece de esta plenitud. Por ende, la familia sustentada en el principio bioético de la justicia, es la responsable de firmar la autorización del internamiento involuntario para proteger al enfermo, al grupo familiar y al entorno social de las consecuencias disruptivas de la enfermedad adictiva. Luego de un adecuado tratamiento, el paciente recupera su plenitud mental, espiritual y física; entonces firma el Consentimiento Informado sin objeción alguna, debido a que ya adquirió conciencia de enfermedad, libre albedrío responsable, autonomía real y sentido de vida. Una vez curado, expresa su gratitud por la oportuna decisión adoptada por sus familiares.

\section{Alternativas}

A continuación, se proponen algunas alternativas para afrontar este problema:

1. Optar por un enfoque científico y humanista del fenómeno adictivo, eliminando las creencias irracionales relativas a las adicciones y sus secuelas.

2. Aceptar que las adicciones constituyen una enfermedad única que comprende las adicciones químicas, tecnológicas, lúdicas y sociales.

3. Reconocer que las adicciones son una enfermedad volitiva recuperable y que comúnmente el adicto carece de conciencia de enfermedad.

4. Proveer a las familias del marco legal que les permita firmar la Autorización de Internamiento Involuntario del adicto indicado por el médico, cuando se trata de adictos avanzados, graves o terminales.

5. Brindar una sólida formación académica en el área de las adicciones, en el pre-grado, post-grado y en la educación continua.

6. Adoptar la política de que las adicciones son un problema de salud pública, cuyo manejo es profesional, especializado, humanista, integral, multimodal y a largo plazo; erradicando a la vez los tratamientos empíricos, meramente mercantilistas.

7. Formar equipos interdisciplinarios de salud con experticia en adicciones, en todos los niveles e instituciones del sector salud, público y privado.

8. Involucrar plena y activamente a la familia como eje de la promoción de la salud mental y espiritual; al igual que en la prevención, tratamiento y rehabilitación de las adicciones, con el apoyo sistemático del equipo profesional especializado.

9. Brindar información completa e idónea a la familia y a la colectividad sobre el fenómeno adictivo.

10. Centrar la promoción y la prevención integral de las adicciones en la comunidad, con énfasis en el binomio familia-centro educativo.

Financiamiento: Autofinanciado.

Conflicto de interés: El autor declara no presentar conflicto de interés en la publicación de este artículo.

Recibido: 01 de Julio de 2016

Aprobado: 08 de Agosto de 2016 


\section{REFERENCIAS BIBLIOGRÁFICAS}

1. Nizama, M. (2003). Guía Para El Manejo Familiar De Las Adicciones. Lima: Fondo Editorial Universidad Alas Peruanas.

2. Rowe, C. L. (2010). Multidimensional Family Therapy: Addressing Co-Occurring Substance Abuse and Other Problems among Adolescents with Comprehensive Family-Based Treatment. Child Adolesc Psychiatr Clin N Am, 19, 563-576. doi: 10.1016/j.chc.2010.03.008.Link: http://www.ncbi. nlm.nih.gov/pmc/articles/PMC2916747/pdf/nihms-189543.pdf.

3. Heru, A. (2006). Family Psychiatry: From Research to Practice. Am J Psychiatry, 163, 962-968. Link: http://ajp.psychiatryonline.org/article.aspx?articleid=96661\&RelatedWidgetArticles=true.

4. Secades, R., Fernández, J. R. (2000). Cómo mejorar las tasas de retención en los tratamientos de drogodependencias. Adicciones, 12, 353-363. Link: http://www.adicciones.es/files/353-363\%200riginal\%204.pdf.

5. American Psychiatric Association. (1994). Diagnostic Criteria from DSMIV, Washington D.C.

6. Asociación Psiquiátrica de América Latina APAL. (2004). Guía Latinoamericana de Diagnóstico Psiquiátrico. Jalisco: Coordinación Editorial Centro Universitario de la Salud Universidad de Guadalajara.

7. Organización Mundial de la Salud. (1994). CIE 10 Glosario de síntomas para los trastornos mentales. Recuperado de: http://whqlibdoc.who.int/ hq/1994/WHO_MNH_MND_94.11_spa.pdf .

8. Iraurgi, I., Sanz, M. y Martínez-Pampliega, A. (2004). Funcionamiento familiar y severidad de los problemas asociados a la adicción a drogas en personas que solicitan tratamiento. Adicciones, 16, 185-195. Link: http:// www.adicciones.es/files/04.\%20lraurgi.pdf.

9. LWork Cover Corporation of South Australia. (2001) Guidelines for drugs, alcohol at the workplace. Recuperado de: http://www.safework. sa.gov.au/contentPages/docs/resDrugAlcoholGuidelines.pdf.

10. Cheng-Fang, Y., Ray, C. H., Ries, R., Shu-Chun, L., Chi-F, H., Yu-Ping, C. y Ming-Lung, Y. (2008). Insight into Alcohol-Related Problems and Its Associations with Severity of Alcohol Consumption, Mental Health Status, Race, and Level of Acculturation in Southern Taiwanese Indigenous People with Alcoholism. Am J Drug Alcohol Abuse, 34, 553-561. doi: 10.1080/00952990802295220. Link: http://www.ph.tcu.edu.tw/file/ apply/apply_master/app_ma_exam/101/101ph\%20indigenous\%20 exam\%20paper/Insight\%20into\%20Alcohol-Related....pdf.

11. Moeller, S., Maloney, T., Parvaz, M., Alia-Klein, N., Woicik, P., Telang, F., Gene-Jack Wang, Volkow N and Goldstein R. (2010). Impaired insight in cocaine addiction: laboratory evidence and effects on cocaine-seeking behavior. Brain, 133, 1484-1493. doi: 10.1093/brain/awq066. Link: http:// brain.oxfordjournals.org/content/133/5/1484.full.pdf+html.

12. Substance Abuse and Mental Health Services Administration (2010). Results from the 2009 National Survey on Drug Use and Health: Volume I. Summary of National findings. Recuperado de: http://www.gmhc.org/ files/editor/file/a_pa_nat_drug_use_survey.pdf.

13. Prochaska, J. (2010). Failure to Treat Tobacco Use in Mental Health and Addiction Treatment Settings: A Form of Harm Reduction? Drug Alcohol Depend. Drug Alcohol Depend, 110, 177-182. Link: http://www.ncbi.nlm. nih.gov/pmc/articles/PMC2916693/pdf/nihms-224315.pdf.
14. Guajardo, H., Kushner, D. (2004). Mayéutica Manual Terapéutico. Santiago de Chile: Editorial de la Universidad de Santiago de Chile.

15. Garrido, M., Jaén, P. y Domínguez, A. (2004). Ludopatía y Relaciones Familiares. Barcelona: Paidós.

16. National Institute of Drug abuse. (2009). Treatment approaches for drug addiction. Recuperado de:http://www.drugabuse.gov/sites/default/ files/if_treatment_approaches_2009_to_nida_92209.pdf.

17. Valle, R. y Perales, A. (2010) Demora en la búsqueda de atención médica en pacientes adictos a sustancias psicoactivas. Adicciones, 22, 73-80. Link: http://www.adicciones.es/files/73-80\%20valle.pdf.

18. Sułek J, Korczak-Dziurdzik A, Korbel-Pawlas M, Lyznicka M, Czarnecki D. (2006). Attitude toward alcoholics and their families. J Physiol Pharmacol, 57, 349-358.Link: t.

19. Grov, C., Kelly, B. C. y Parsons, J. T. (2009). Polydrug Use Among ClubGoing Young Adults Recruited Through Time-Space Sampling. Substance Use \& Misuse, 44, 848-864. doi: 10.1080/10826080802484702. Link: http:// www.ncbi.nlm.nih.gov/pmc/articles/PMC2683356/.

20. Kaplan, H. y Sadocks, B. (2003). Synopsis of Psychiatry: Behavioral Sciences, Clinical Psychiatry (pp. 88-107). (9th ed). Philadelfia: Lippincott Williams \& Wilkins.

21. Kupfermann, I., Kandel, E. R. y Iversen, S. Kandel, E.R; Schwartz, J.H y Jessell, T. M. (2001). Estados de Motivación y Adictivos. En: Principios de Neurociencia. (pp. 998-1014) (4th ed). Madrid: McGraW-Hill.

22. Goldstein R, Volkow N. (2002). Drug Addiction and Its Underlying Neurobiological Basis: Neuroimaging Evidence for the Involvement of the Frontal Cortex. Am J Psychiatry, 159, 1642-1652. Link: http://ajp.psychiatryonline.org/data/Journals/AJP/3741/1642.pdf.

23. Nizama, M. (2009). Tratamiento Familiar Holístico de las adicciones. Lima: CENTRO DE Producción Editorial e Imprenta de la Universidad Nacional Mayor de San Marcos.

24. Nizama-Vía, A. y Nizama-Valladolid, M. (2012). Manejo empírico de las adicciones en el Perú [carta al Editor]. Rev Perú Med Exp Salud Publica, 29, 149-67.Link: http://www.scielo.org.pe/pdf/rins/v29n1/a36v29n1.pdf

25. Fiestas, F., Ponce, J. (2011). Eficacia de comunidades terapéuticas en el tratamiento de problemas relacionados con uso de sustancias psicoactivas: una revisión sistemática. Recuperado de: http://www.ins.gob.pe/repositorioaps/0/4/jer/evidencias/Nota\%20t\%C3\%A9cnica-2_Eficacia\%20 de $\% 20$ comunidades $\% 20$ terap $\%$ C3\%A9uticas $\% 20$ en $\% 20$ el\%20tratamiento\%20sustancias\%20psicoactivas\%20una\%20revisi\%C3\%B3n\%20 sistem\%C3\%A1tica.pdf.

26. Colegio Médico del Perú. (2007). Código de Ética y Deontología. Recuperado de: http://www.cmp.org.pe/doc_norm/codigo_etica_cmp_OCT2007.pdf.

27. Morera B. (2000). Aspectos bioéticos de la asistencia al drogodependiente. adicciones, 12, 515-526. Link: http://www.adicciones.es/ files/515-526\%200riginal.pdf28. Aithal A. P. The impact of physical activities on the academic performance of medical students. Online Journal of Health and Allied Sciences. 2016; 15(2). 